\title{
Comparison of the free and bound phenolic profiles and cellular antioxidant activities of litchi pulp extracts from different solvents
}

Dongxiao Su ${ }^{1,2 \dagger}$, Ruifen Zhang ${ }^{2 \dagger}$, Fangli Hou ${ }^{3}$, Mingwei Zhang ${ }^{2 *}$, Jinxin Guo ${ }^{2}$, Fei Huang ${ }^{1,2}$, Yuanyuan Deng ${ }^{2}$ and Zhencheng $\mathrm{Wei}^{2}$

\begin{abstract}
Background: The phenolic contents and antioxidant activities of fruits could be underestimated if the bound phenolic compounds are not considered. In the present study, the extraction efficiencies of various solvents were investigated in terms of the total content of the free and bound phenolic compounds, as well as the phenolic profiles and antioxidant activities of the extracts.

Methods: Five different solvent mixtures were used to extract the free phenolic compounds from litchi pulp. Alkaline and acidic hydrolysis methods were compared for the hydrolysis of bound phenolic compounds from litchi pulp residue. The phenolic compositions of the free and bound fractions from the litchi pulp were identified using HPLC-DAD. The antioxidant activities of the litchi pulp extracts were determined by oxygen radical absorbance capacity (ORAC) and cellular antioxidant activity (CAA) assays.

Results: Of the solvents tested, aqueous acetone extracted the largest amount of total free phenolic compounds (210.7 mg GAE/100 g FW) from litchi pulp, followed sequentially by aqueous mixtures of methanol, ethanol and ethyl acetate, and water itself. The acid hydrolysis method released twice as many bound phenolic compounds as the alkaline hydrolysis method. Nine phenolic compounds were detected in the aqueous acetone extract. In contrast, not all of these compounds were found in the other four extracts. The classification and content of the bound phenolic compounds released by the acid hydrolysis method were higher than those achieved by the alkaline hydrolysis. The aqueous acetone extract showing the highest ORAC value (3406.9 $\mu \mathrm{mol}$ TE/100 $\mathrm{g}$ FW) for the free phenolic extracts. For the CAA method, however, the aqueous acetone and methanol extracts (56.7 and $55.1 \mu \mathrm{mol} \mathrm{QE} / 100 \mathrm{~g} \mathrm{FW}$ ) showed the highest levels of activity of the five extracts tested. The ORAC and CAA values of the bound phenolic compounds obtained by acid hydrolysis were 2.6- and 1.9-fold higher than those obtained using the alkaline hydrolysis method.

Conclusions: The free and bound phenolic contents and profiles and antioxidant activities of the extracts were found to be dependent on the extraction solvent used. Litchi exhibited good cellular antioxidant activity and could be a potentially useful natural source of antioxidants.
\end{abstract}

\footnotetext{
* Correspondence: mwzhh@vip.tom.com

${ }^{\dagger}$ Equal contributors

${ }^{2}$ Sericultural and Agri-food Research Institute, Guangdong Academy of

Agricultural Sciences, Guangzhou 510610, P.R. China

Full list of author information is available at the end of the article
} 


\section{Background}

The regular consumption of fruits and vegetables has long been associated with the prevention of many chronic diseases, including cardiovascular disease and cancer, as evidenced by the results of clinical trials and epidemiological studies [1]. Oxidative stress is believed to be an important contributing factor in the development of these diseases, and the potent antioxidant properties of phytochemicals, including the phenolic compounds found in fruits and vegetables especially, may help to prevent the effects of oxidative stress on the body [2].

Litchi (Litchi chinensis Sonn.), which is a subtropical fruit, is fast becoming popular throughout the world because of its attractive appearance and delicious taste [3]. Although the phenolic constituents in the pericarp and seed of litchi have been well characterized [4-6], very few studies have been reported pertaining to the phenolic profiles of litchi pulp extracts in terms of their potential health benefits.

It is well known that phenolic compounds exist in both free and bound forms in plant cells, and that the free phenolic compounds are solvent extractable. In contrast, the bound phenolic compounds, which are covalently bound to the plant matrix, cannot be extracted into water or aqueous/organic solvents mixtures [7]. Although the total phenolic contents and antioxidant activities of litchi pulp have been reported previously [8-10], these studies only considered the solvent extractable free phenolic compounds present in the pulp. J Sun, YF Chu, Xz Wu and RH Liu [11] reported that about 4$57 \%$ of the phenolic compounds present in fruits existed in their bound forms. With this in mind, the phenolic contents and antioxidant activities of different fruits could well be underestimated, to a large extent, if the bound fractions are not considered in some way.

Furthermore, the extraction procedure used for the isolation of the phenolic compounds from plant materials could have a significant impact of the outcome of any investigation aimed at evaluating the properties of the phenolic compounds in plant materials, because it would dictate the nature and quantity of the phenolic compounds obtained in the extracts [12]. During their investigation of the extractive capability of various aqueous solvent systems (i.e., methanol, acetone and chloroform) A Ghasemzadeh, HZ Jaafar and A Rahmat [13] found that aqueous methanol afforded the highest level of phenolic and flavonoid compounds from the leaves, stems and rhizomes of two young ginger varieties. A higher phenolic extraction efficiency could be realized with an aqueous ethanol or methanol system, whereas the specific extraction of flavan-3-ols and proanthocyanidins could be achieved using acetone-based mixtures [14]. It is therefore important to select the most appropriate solvent for the extraction of phenolic compounds to allow for a high level of extraction efficiency. Furthermore, the bound phenolic compounds are covalently conjugated to cellulose, pectin and polysaccharides through ester bonds, and can be difficult to hydrolyze $[15,16]$. Alkaline, acidic or enzymatic hydrolysis methods can be used to release bound phenolic compound. In most of the studies conducted in this area, the bound phenolic compounds were released by alkaline hydrolysis, including, for example, our own research towards the preparation of litchi bound phenolic compounds [11,17-19]. In contrast, Bonoli et al. [14] reported the use of acid hydrolysis, and claimed that this method was more effective for the release of bound phenolic compounds than alkaline hydrolysis. In our previous study, we demonstrated that litchi pulp contained a high content of total phenolic and flavonoid compounds, although the procedure used for the extraction of the free and bound phenolic compounds in this particular study was based on a series of methods designed specifically for the extraction of other unrelated materials [19]. Thus, it still remains unclear as to what the most efficient solvent system would be for the extraction of free and bound phenolic compounds from litchi pulp.

Chemical antioxidant activity assays, such as ferric reducing/antioxidant power (FRAP) and oxygen radical absorbance capacity (ORAC), are commonly used in food studies, but these methods cannot account for biological activity. Biological systems are considerably more complex than simple chemical mixtures, and antioxidants may operate via multiple mechanisms [20]. The ability of chemical antioxidant activity assays to predict in vivo activity has been called into question on several occasions because none of these methods take account of the bioavailability, uptake and metabolism of the antioxidants. As a model based on cell culture, cellular antioxidant activity (CAA) represents a more biologically relevant method for the determination of antioxidant activity than the more commonly used "test tube" chemistry methods [2]. Using CAA to estimate the antioxidant activity of litchi pulp could therefore enhance our knowledge and understanding of the biological activity of litchi pulp.

The aim of the current investigation was to compare the differences in the extracts of litchi pulp, in terms of their total phenolic contents, phenolic profiles and antioxidant activities, when the free phenolic compounds had been extracted with different solvent systems and the bound phenolic compounds had been released using acid and alkaline hydrolysis methods. Using these results, it would then be possible to select appropriate methods for the extraction of free and bound phenolic compounds from litchi pulp.

\section{Methods \\ Materials}

The variety Feizixiao, which is one of the main litchi cultivars in South China, was purchased from a local fruit 
market in Guangzhou, China. The litchi fruit was carefully examined and identified by Professor Liangxi Ou, South China fruit germplasm resources evaluation scientist of Fruit Tree Research Institute, Guangdong Academy of Agricultural Sciences. A voucher specimen (NO 20120515) has been preserved in the refrigeration house $\left(-20^{\circ} \mathrm{C}\right)$, Faculty of Horticulture, South China Agricultural University. Fully ripened fruits with brightly coloured red skins were selected, washed, peeled and de-seeded. Approximately $150 \mathrm{~g}$ of litchi pulp was used for each treatment.

\section{Chemicals}

Gallic acid, chlorogenic acid, (+)-catechin hydrate, vanillic acid, caffeic acid, syringic acid, epicatechin, 4-methylcatechol, coumarin, ferulic acid, resveratrol, quercetin, 3,4-dihydroxybenzoic acid, $p$-coumaric acid, rutin, sinapic acid, 6-hydroxy-2,5,7,8-tetramethylchroman-2-carboxylic acid (Trolox), Folin-Ciocalteu's phenol reagent, 2',7'-dichlorofluorescin diacetate (DCFH-DA), fluorescein disodium salt, 2,2' -azobis (2-amidinopropane) dihydrochloride (ABAP) and quercetin dehydrate were purchased from SigmaAldrich (St. Louis, MO, USA). Dulbecco's Modified Eagle's Medium (DMEM), dimethyl sulphoxide, HPLC-grade acetic acid and acetonitrile were obtained from Thermo Fisher Scientific (Waltham, MA, USA). Human liver cancer cells (HepG2 cells) were obtained from the American Type Culture Collection (ATCC) (Rockville, MD, USA). Fetal bovine serum (FBS) was obtained from Haoyang Biologicals (Tianjin, China). Deionised water was prepared using a Milli-Q water purification system (Billerica, MA, USA).

\section{Extraction of free phenolic compounds from litchi pulp}

The free phenolic compounds were extracted according to a published procedure [11]. Briefly, $150 \mathrm{~g}$ of freshly prepared litchi pulp was blended for $5 \mathrm{~min}$ in chilled solvent (80\% methanol, $80 \%$ ethanol, $80 \%$ acetone, $80 \%$ ethyl acetate or deionised water; $1: 2$, w/v) using a Philips blender (Philips, Zhuhai, China). The samples were then homogenised using a XHF-D homogeniser (Ningbo Xinzhi-Bio Technology Co. Ltd., Ningbo, China) at $5000 \mathrm{rpm}$ for $5 \mathrm{~min}$ at $4^{\circ} \mathrm{C}$. The homogenates were centrifuged at $5000 \mathrm{rpm}$ for $8 \mathrm{~min}$ (Changsha Xiangzhi Instrument Co. Ltd., Changsha, China). The pellets were re-extracted with $300 \mathrm{~mL}$ of the original solvent and the supernatants were combined and evaporated to near dryness in vacuo on a rotary evaporator at $45^{\circ} \mathrm{C}$. The concentrated supernatant was then reconstituted with methanol/water $(85: 15, \mathrm{v} / \mathrm{v})$ and stored at $-80^{\circ} \mathrm{C}$. For the water extraction, the samples were extracted on a rocker incubator (Shanghai Yuejin Medical Instrument Factory, Shanghai, China) at room temperature for $120 \mathrm{~min}$.
Extraction of bound phenolic compounds from litchi pulp The bound phenolic compounds were extracted from the residue resulting from the free phenolic extraction using $80 \%$ acetone. Six portions of the residue were prepared for the determination of the bound phenolic compounds in the litchi pulp.

\section{Alkaline hydrolysis method}

The bound phenolic compounds in the litchi samples were extracted using a modified version of the alkaline hydrolysis method reported by $\mathrm{M}$ Bonoli, V Verardo, E Marconi and MF Caboni [14]. Briefly, the residue was digested in $2 \mathrm{M}$ sodium hydroxide and stirred at room temperature for $18 \mathrm{~h}$ with shaking under nitrogen gas. The mixture was then neutralised with concentrated hydrochloric acid before being extracted six times with ethyl acetate. The organic fractions were combined and evaporated to dryness in vacuo at $35^{\circ} \mathrm{C}$. The phenolic compounds were reconstituted in methanol/water $(1 \mathrm{M}$; $85: 15, \mathrm{v} / \mathrm{v}$ ) and stored at $-80^{\circ} \mathrm{C}$.

\section{Acid hydrolysis method}

The bound phenolic compounds in the litchi pulp were extracted with acid hydrolysis method according to a previously described method [21]. Briefly, $30 \mathrm{~g}$ samples of litchi residue were treated with $120 \mathrm{~mL}$ of methanol/ $\mathrm{H}_{2} \mathrm{SO}_{4}(90: 10, \mathrm{v} / \mathrm{v})$ at $85^{\circ} \mathrm{C}$ for $20 \mathrm{~h}$, and the resulting mixtures were neutralised with $10 \mathrm{M}$ sodium hydroxide before being extracted six times with ethyl acetate. The organic fractions were combined and evaporated to dryness in vacuo at $35^{\circ} \mathrm{C}$ before being reconstituted with methanol/water $(85: 15, \mathrm{v} / \mathrm{v})$ and stored at $-80^{\circ} \mathrm{C}$.

\section{Determination of the phenolic contents in litchi pulp Total phenolic contents}

The total phenolic contents were determined using the Folin-Ciocalteu (FC) colourimetric method described by Dewanto et al. [22]. Briefly, an aliquot $(125 \mu \mathrm{L})$ of each of the extracts described above or a standard solution was mixed with $0.5 \mathrm{~mL}$ of deionised water and $125 \mu \mathrm{L}$ of the FC reagent. After $6 \mathrm{~min}, 1.25 \mathrm{~mL}$ of a $7 \% \mathrm{Na}_{2} \mathrm{CO}_{3}$ solution was added to the mixture, followed by $1.0 \mathrm{~mL}$ of water to bring the final volume to $3.0 \mathrm{~mL}$. After 90 min of incubation at ambient temperature in the absence of light, the absorbance at $760 \mathrm{~nm}$ was measured using a Shimadzu UV-1800 spectrometer (Shimadzu Inc., Kyoto, Japan). Gallic acid was used as the standard, and the total phenolic contents were expressed as mg gallic acid equivalents (GAE)/100 g FW.

\section{Total flavonoid contents}

The total flavonoid contents were determined using the method reported by Dewanto et al. [22]. Briefly, an aliquot $(250 \mu \mathrm{L})$ of each extract or a standard solution was 
mixed with $1.25 \mathrm{~mL}$ of deionised water followed by $75 \mu \mathrm{L}$ of a $5 \% \mathrm{NaNO}_{2}$ solution. After $6 \mathrm{~min}, 150 \mu \mathrm{L}$ of a $10 \% \mathrm{AlCl}_{3} \cdot 6 \mathrm{H}_{2} \mathrm{O}$ solution was added to each mixture. After $5 \mathrm{~min}, 0.5 \mathrm{~mL}$ of $1 \mathrm{M} \mathrm{NaOH}$ was added, and the total volume was adjusted to $3.0 \mathrm{~mL}$ with deionised water. (+)-Catechin was used as a standard. The absorbance at $510 \mathrm{~nm}$, which was corrected using a blank, was then determined and the results were expressed as mg of (+)-catechin equivalents (CE)/100 g FW.

\section{Tannin contents}

The tannin contents of the litchi pulp were determined using the method reported by Julkunen-Titto [23]. An aliquot $(200 \mu \mathrm{L})$ of each extract or the standard solution was mixed with $3.0 \mathrm{~mL}$ of a 1:1 (v/v) mixture of $4 \%$ vanillin in methanol and $30 \% \mathrm{H}_{2} \mathrm{SO}_{4}$ in methanol, and the resulting mixture was incubated for $20 \mathrm{~min}$ at room temperature in the absence of light. The absorbance at $510 \mathrm{~nm}$ was then read using a Shimadzu UV-1800 spectrometer. (+)-Catechin was used as a standard. The contents of the condensed tannin in the free fraction and the hydrolysed tannin in the bound fraction of the litchi pulp were expressed as $\mathrm{mg}$ of (+)-catechin equivalents (CE)/100 g FW.

\section{Analysis of phenolic compound compositions in litchi pulp} The phenolic compositions of the litchi pulp samples were determined using an HPLC-DAD method [19]. The HPLC separations were performed on an Agilent Zorbox SB-C 18 column $(250 \times 4.6 \mathrm{~mm}, 5 \mu \mathrm{m}$, Palo Alto, CA, USA) at a flow rate of $1.0 \mathrm{~mL} / \mathrm{min}$ using an injection volume of $20 \mu \mathrm{L}$. The binary gradient consisted of solution A (water/acetic acid 996:4 v/v) and solution B (acetonitrile). The gradient conditions were as follows: 0-40 min, solution B 5-25\%; 40-45 min, solution B 25$35 \%$; and 45-50 min, solution B 35-50\%, followed by a 5 min equilibration period with $5 \%$ solution B. Each injection was monitored at $280 \mathrm{~nm}$. The identity of each peak was confirmed based on the retention time determined for each pure compound. Values are expressed as $\mu \mathrm{g} / 100 \mathrm{~g}$ FW.

\section{Assay of antioxidant capacity of litchi pulp Determination of ORAC}

The ORAC was performed according to the method described by Wolfe et al. [24]. The phenolic extracts were diluted with $75 \mathrm{mM}$ phosphate buffer $(\mathrm{pH}=7.4)$. The assay was performed in black-walled 96-well plates (Corning Scientific, Corning, NY, USA). Each well contained $20 \mu \mathrm{L}$ of the extract or $20 \mu \mathrm{L}$ of the trolox standard (range, 6.25-50 $\mu \mathrm{M}$ ) or a blank and $200 \mu \mathrm{L}$ of fluorescein (final concentration of $0.96 \mu \mathrm{M}$ ). The plates were incubated at $37^{\circ} \mathrm{C}$ for $20 \mathrm{~min}$ on an Infinite M200pro plate reader (Tecan Austria GmbH, Salzburg,
Austria). After incubation, $20 \mu \mathrm{L}$ of $119 \mathrm{mM}$ ABAP, which was freshly prepared for each run, was added to each well except the F well, which was treated with $20 \mu \mathrm{L}$ of $75 \mathrm{mM}$ phosphate buffer. The fluorescence conditions were as follows: excitation at $485 \mathrm{~nm}$ and emission at $520 \mathrm{~nm}$ for 35 cycles at intervals of $4.5 \mathrm{~min}$. The ORAC results were expressed as $\mu \mathrm{mol}$ trolox equivalents (TE)/100 g FW.

\section{Measurement of CAA}

The CAA assay was conducted according to the method described by Wolfe et al. [24]. Briefly, HepG2 cells were seeded at a density of $6 \times 10^{4} /$ well in a black 96 -well microplate in $100 \mu \mathrm{L}$ of growth medium (DMEM medium containing $10 \%$ fetal bovine serum). The growth medium was removed after $24 \mathrm{~h}$, and the triplicate wells were then treated for $1 \mathrm{~h}$ with $100 \mu \mathrm{L}$ portions of different concentration of quercetin or the litchi pulp extracts plus $25 \mu \mathrm{M}$ DCFH-DA in DMEM. The cells were then treated with $100 \mu \mathrm{L}$ of $600 \mu \mathrm{M}$ ABAP in PBS, and the fluorescence was measured with an Infinite M200pro plate reader using an excitation wavelength of $485 \mathrm{~nm}$ and an emission wavelength of $520 \mathrm{~nm}$ for 12 cycles at 5 min intervals. The CAA values were calculated according to the method of Wolfe et al. [24]. The CAA results are reported as micromoles of quercetin equivalents (QE) per $100 \mathrm{~g}$ of lychee pulp FW.

\section{Statistical analyses}

All of the results are reported as the mean \pm SD for triplicate determinations of each sample. The results of the acid and alkaline hydrolyses were analysed using the $t$ test. A one-way ANOVA test was used, followed by an SNK-q test, to compare the extracts obtained from the different solvents. A value of $p<0.05$ was considered statistically significant. All of the statistical analyses were performed using SPSS statistical package version 13.0 software (SPSS Inc. Chicago, IL, USA).

\section{Results}

Effects of the different solvent extraction on the content of free and bound phenolic compounds in litchi pulp

The contents of the free and bound phenolic, flavonoid and tannin compounds in the litchi pulp are shown in Tables 1 and 2 . Aqueous acetone gave the highest yield for the extraction of the free phenolic, flavonoid and condensed tannin compounds, followed by aqueous mixtures of methanol, ethanol and ethyl acetate $(p<0.05)$. The water extract afforded the lowest contents for the different classes of phenolic compounds. The flavonoids content in the $80 \%$ aqueous methanol extract was not significantly different from that of the aqueous ethanol extract.

For the bound phenolic compounds, the results revealed that the acid hydrolysis method led to the release 
Table 1 Contents of the free phenolic, flavonoid and tannin compounds of the litchi pulp in various solvents extracts

\begin{tabular}{lccccc}
\hline & \multicolumn{4}{c}{ Free phenolic compounds } \\
\cline { 2 - 6 } & $\mathbf{8 0 \%}$ methanol & $\mathbf{8 0 \%}$ ethanol & $\mathbf{8 0 \%}$ acetone & $\mathbf{8 0 \%}$ ethyl acetate & Water \\
\hline Phenolic (mg GAE/100 g FW) & $190.69 \pm 3.69 \mathrm{~b}^{1}$ & $171.81 \pm 3.42 \mathrm{C}$ & $210.67 \pm 9.85 \mathrm{a}$ & $151.79 \pm 5.70 \mathrm{~d}$ & $121.76 \pm 4.23 \mathrm{e}$ \\
Flavonoid (mg CE/100 g FW) & $85.55 \pm 2.34 \mathrm{~b}$ & $80.84 \pm 2.98 \mathrm{~b}$ & $103.88 \pm 1.82 \mathrm{a}$ & $65.17 \pm 1.7 \mathrm{C}$ & $57.15 \pm 1.31 \mathrm{~d}$ \\
Tannin (mg CE/100 g FW) & $114.32 \pm 3.33 \mathrm{~b}$ & $107.02 \pm 1.45 \mathrm{c}$ & $156.08 \pm 2.85 \mathrm{a}$ & $80.98 \pm 2.69 \mathrm{~d}$ & $70.93 \pm 1.98 \mathrm{e}$ \\
\hline
\end{tabular}

'Values not sharing a common letter in each row are significantly different $(p<0.05)$.

of far more bound phenolic compounds from the residue of litchi pulp than the alkaline hydrolysis, where free phenolic compounds had been extracted with $80 \%$ acetone $(p<0.05)$. The contents of the phenolic, flavonoid and hydrolysed tannin compounds in the extract from the acid hydrolysis were determined to be 2.0, 1.6, and 2.3-fold greater than those in alkaline hydrolysis extract, respectively.

\section{Effects of different solvents on the composition of free} and bound phenolic compounds in litchi pulp

Analysis by HPLC-DAD revealed that ten phenolic compounds were present in the litchi pulp extracts, including 3,4-dihydroxybenzoic acid, (+)-catechin, vanillic acid, caffeic acid, syringic acid, (-)-epicatechin, 4-methylcatechol, ferulic acid, rutin and quercetin (Tables 3 and 4). The number and type of phenolic compounds detected in the litchi pulp extracts obtained from different extraction solvents varied considerable (Table 3). All the phenolic compounds mentioned above were detected in the free forms from the litchi pulp extract obtained using $80 \%$ acetone except quercetin, which was not detected in the free form in any of the five solvent systems. In contrast, only seven of the ten phenolic compounds described above were detected in the extracts obtained from the other four solvents systems. 3,4-Dihydroxybenzoic acid was not extracted in any of these four solvent systems and syringic acid was not extracted when $80 \%$ methanol, $80 \%$ ethyl acetate or water was used as the extraction solvent. Although similar contents of syringic acid were detected in the litchi pulp extracts obtained when $80 \%$ acetone and $80 \%$ ethanol were used as the extraction solvents, vanillic acid was not detected in the latter of these two systems. Besides, the five extraction solvents exhibited significantly

Table 2 Contents of the bound phenolic, flavonoid and tannin compounds of the litchi pulp in various hydrolysis extracts

\begin{tabular}{lcc}
\hline & \multicolumn{2}{c}{ Bound phenolic compounds } \\
\cline { 2 - 3 } & Acid hydrolysis & Alkaline hydrolysis \\
\hline Phenolic (mg GAE/100 g FW) & $61.27 \pm 1.78^{* * 1}$ & $30.71 \pm 0.51$ \\
Flavonoid (mg CE/100 g FW) & $29.74 \pm 1.44^{* *}$ & $18.16 \pm 0.83$ \\
Tannin (mg CE/100 g FW) & $37.37 \pm 3.49^{* *}$ & $15.86 \pm 1.70$ \\
\hline
\end{tabular}

${ }^{1}$ Asterisk $\left(^{* *}\right)$ within the same row indicate significant difference $(p<0.01)$. different extraction efficiencies for the nine compounds. As shown in Table 3, 4-methylcatechol, rutin and (-)-epicatechin were determined to be the major phenolic compounds extracted in their free form the litchi pulp in all five solvent systems. The highest 4-methylcatechol content was found in extracts obtained using the $80 \%$ acetone and $80 \%$ methanol solvent systems, followed by $80 \%$ ethanol, $80 \%$ Ethyl acetate and water. The latter two had similar extraction efficiencies for 4-methylcatechol $(p>0.05)$. Aqueous acetone afforded a 4-methylcatechol yield that was almost 2 -fold greater than that of water $(p<0.05)$. The second most abundant phenolic compound extracted from the litchi pulp was rutin. The ranking order for the rutin yields given by the five different solvent systems was similar to that of 4-methylcatechol. The extraction efficiency of aqueous acetone for (-)-epicatechin was significantly higher than that of methanol, follow sequentially by $80 \%$ ethanol, $80 \%$ Ethyl acetate and water $(p<0.05)$. The use of $80 \%$ acetone allowed for the extraction of $0.5-, 1.1-$, 1.5 - and 7.7-fold more (-)-epicatechin than $80 \%$ methanol, $80 \%$ ethanol, $80 \%$ ethyl acetate and water, respectively. The concentrations of the other phenolic components were less than $500 \mu \mathrm{g} / 100 \mathrm{~g}$ FW. The orders of the extraction efficiencies for other phenolic compounds were similar except for water, which had the highest extraction efficiency for vanillic acid of all of the solvent systems, followed sequentially by acetone, methanol and ethyl acetate. As mentioned above, the $80 \%$ ethanol solvent system did not extract vanillic acid from litchi pulp.

The types and quantities of the bound phenolic compounds released from the litchi pulp were influenced by the hydrolysis method (Table 4). Seven phenolic components were found in the extract following the acid hydrolysis of the litchi pulp residue, whereas only four phenolic compounds were found in the extract following alkaline hydrolysis. (-)-Epicatechin, rutin and quercetin were released by both the acid and alkaline hydrolyses. Four phenolic compounds, including vanillic acid, caffeic acid, syringic acid and 4-methylcatechol, were detected exclusively in the extracts resulting from the acid hydrolysis, whereas $(+)$-catechin was only found in extracts following the alkaline hydrolysis. The quantitative analysis of the extracts following the acid and alkaline hydrolysis treatments showed that 4-methylcatechol and (-)-epicatechin were the main phenolic components 
Table 3 Free phenolic profiles of litchi pulp

\begin{tabular}{lccccc}
\hline Phenolic & \multicolumn{4}{c}{ Free phenolic compounds } \\
\cline { 2 - 6 } & $\mathbf{8 0 \%}$ methanol & $\mathbf{8 0 \%}$ ethanol & $\mathbf{8 0 \%}$ acetone & $\mathbf{8 0 \%}$ ethyl acetate & Water \\
\hline 3,4-dihydroxybenzoic acid & $\mathrm{ND}^{1}$ & $\mathrm{ND}$ & $115.26 \pm 11.17^{2}$ & $\mathrm{ND}$ & $\mathrm{ND}$ \\
(+)-catechin & $140.34 \pm 12.01 \mathrm{ab}$ & $131.66 \pm 2.74 \mathrm{bc}$ & $163.34 \pm 21.13 \mathrm{a}$ & $83.34 \pm 5.27 \mathrm{~d}$ & $107.78 \pm 25.53 \mathrm{~cd}$ \\
Vanillic acid & $130.77 \pm 19.50 \mathrm{~b}$ & $\mathrm{ND}$ & $126.48 \pm 7.27 \mathrm{~b}$ & $72.99 \pm 4.90 \mathrm{c}$ & $254.25 \pm 32.88 \mathrm{a}$ \\
Caffeic acid & $393.37 \pm 21.08 \mathrm{~b}$ & $188.95 \pm 16.28 \mathrm{~d}$ & $442.93 \pm 17.78 \mathrm{a}$ & $244.68 \pm 11.43 \mathrm{C}$ & $38.66 \pm 4.57 \mathrm{e}$ \\
Syringic acid & $\mathrm{ND}$ & $30.55 \pm 1.48$ & $39.50 \pm 6.17$ & $\mathrm{ND}$ & $\mathrm{ND}$ \\
(-)-epicatechin & $1489.88 \pm 159.63 \mathrm{~b}$ & $1118.81 \pm 20.54 \mathrm{c}$ & $2308.19 \pm 106.54 \mathrm{a}$ & $929.13 \pm 18.89 \mathrm{~d}$ & $266.28 \pm 13.99 \mathrm{e}$ \\
4-methylcatechol & $5926.88 \pm 475.53 \mathrm{a}$ & $4938.91 \pm 483.11 \mathrm{~b}$ & $6337.70 \pm 209.91 \mathrm{a}$ & $3493.87 \pm 236.51 \mathrm{C}$ & $3304.28 \pm 148.94 \mathrm{c}$ \\
Ferulic acid & $442.64 \pm 19.31 \mathrm{a}$ & $276.60 \pm 21.05 \mathrm{~b}$ & $488.34 \pm 68.82 \mathrm{a}$ & $286.17 \pm 86.28 \mathrm{~b}$ & $215.51 \pm 13.89 \mathrm{~b}$ \\
Rutin & $3441.47 \pm 212.65 \mathrm{ab}$ & $3125.16 \pm 94.31 \mathrm{~b}$ & $3580.33 \pm 274.25 \mathrm{a}$ & $2016.82 \pm 108.41 \mathrm{C}$ & $1614.32 \pm 120.93 \mathrm{~d}$ \\
\hline
\end{tabular}

${ }^{1} \mathrm{ND}$ - not detected.

${ }^{2}$ Values expressed as $\mu \mathrm{g} / 100 \mathrm{~g}$ FW.

${ }^{3}$ Values not sharing a common letter within the same row are significantly different $(p<0.05)$.

detected in the bound fractions of litchi pulp when the residues were extracted by acid or alkaline hydrolysis, respectively.

\section{Antioxidant capacity of litchi pulp extracts from different solvents extraction}

The antioxidant activities of the litchi pulp extracts were evaluated using the ORAC and CAA assays. The antioxidant activity determined by ORAC for the free fraction is shown in Figure 1. The extracts obtained from the five different solvent systems showed significantly different ORAC values $(p<0.05)$ in the range of $1075.66 \pm 129.88$ to $3406.95 \pm 176.14 \mu \mathrm{mol} \mathrm{TE} / 100 \mathrm{~g}$ FW. The aqueous acetone extract had the highest antioxidant activity, followed sequentially by the methanol $(2971.96 \pm 187.01 \mu \mathrm{mol}$ TE/100 g FW), ethanol $(2461.09 \pm 178.35 \mu \mathrm{mol}$ TE/100 g FW) and ethyl acetate extracts $(1433.08 \pm 123.54 \mu \mathrm{mol}$ TE/100 g FW). The lowest ORAC antioxidant activity was

Table 4 Bound phenolic profiles of litchi pulp

\begin{tabular}{|c|c|c|}
\hline \multirow[t]{2}{*}{ Phenolic } & \multicolumn{2}{|c|}{ Bound phenolic compounds ${ }^{b}$} \\
\hline & Alkaline hydrolysis & Acid hydrolysis \\
\hline$(+)$-catechin & $14.02 \pm 3.99^{1}$ & $\mathrm{ND}^{2}$ \\
\hline Vanillic acid & ND & $37.10 \pm 4.88$ \\
\hline Caffeic acid & ND & $95.52 \pm 2.44$ \\
\hline Syringic acid & ND & $4.98 \pm 1.36$ \\
\hline (-)-epicatechin & $207.95 \pm 23.70^{* * 3}$ & $50.57 \pm 6.32$ \\
\hline 4-methylcatechol & ND & $2404.97 \pm 216.36$ \\
\hline Rutin & $83.11 \pm 10.99^{* *}$ & $13.08 \pm 0.96$ \\
\hline Quercetin & $28.77 \pm 10.38$ & $56.12 \pm 7.73^{*}$ \\
\hline
\end{tabular}

detected in the water extract. The ORAC value of the $80 \%$ acetone extract was higher than that of the $80 \%$ methanol extract by approximately $15 \%$ and was more than 2 -fold higher than that of the water extract. The CAA values (Figure 2) of the acetone and methanol extracts, however, were equally the greatest $(56.73 \pm 3.53$ and $55.13 \pm$ $5.03 \mu \mathrm{mol}$ QE/100 g FW, respectively) $(p>0.05)$. The activities of the solvent systems were of the same order as those observed in the ORAC assay, ranking as ethanol $(41.77 \pm 2.29 \mu \mathrm{mol} \mathrm{QE} / 100 \mathrm{~g} \mathrm{FW})>$ ethyl acetate $(32.78 \pm$ $7.11 \mu \mathrm{mol}$ QE/100 g FW) > water $(25.35 \pm 3.22 \mu \mathrm{mol} \mathrm{QE} /$ $100 \mathrm{~g}$ FW).

The CAA and ORAC values $(7.40 \pm 1.49 \mu \mathrm{mol} Q E / 100 \mathrm{~g}$ FW and 286.14 $\pm 45.63 \mu \mathrm{mol}$ TE/100 g FW) (Figure 3) for the bound phenolic compounds released by acid hydrolysis were 1.9 and 2.6-fold higher than those obtained by alkaline hydrolysis $(3.95 \pm 0.89 \mu \mathrm{mol} \mathrm{QE} / 100 \mathrm{~g} \mathrm{FW}$ and $110.16 \pm 8.43 \mu \mathrm{mol} \mathrm{TE} / 100 \mathrm{~g}$ FW $)(p<0.05)$.

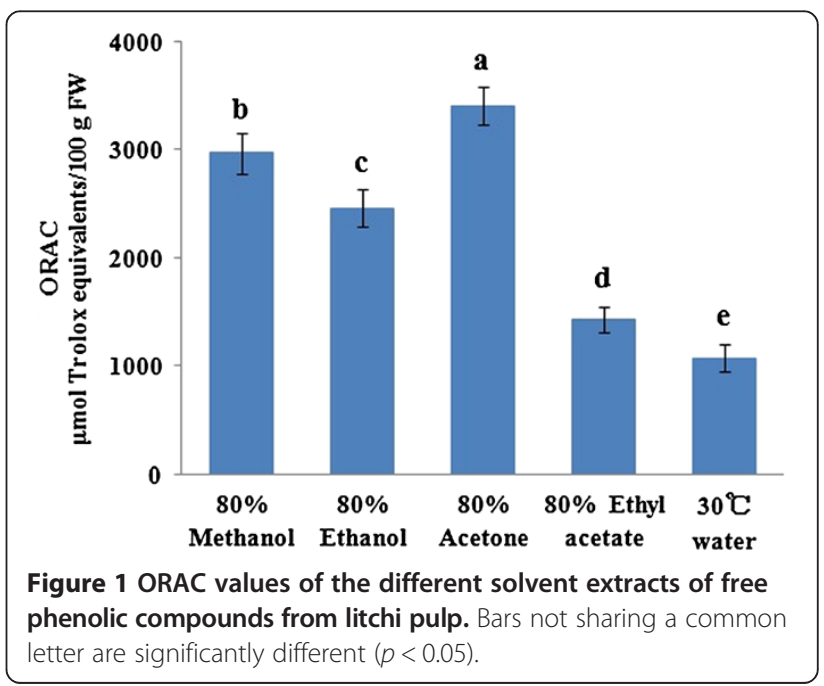




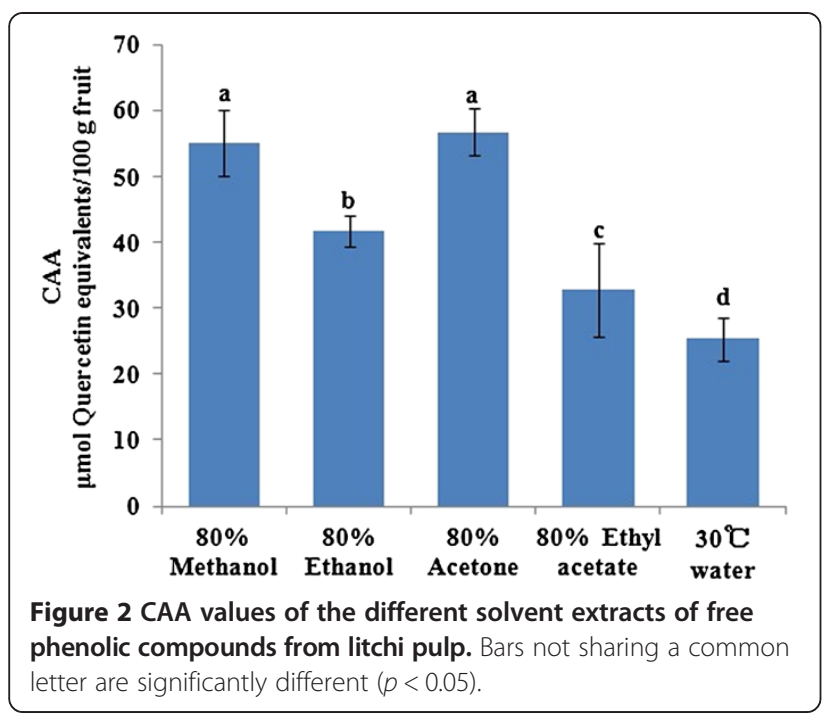

\section{Discussion}

Effects of extraction solvents on the contents of free and bound phenolic compounds in litchi pulp extracts

The choice of solvent for the extraction of the polyphenolic compounds from plant materials is greatly important because different solvents influence the extraction efficiencies of the different phenolic components in different ways. The results of the current study revealed that $80 \%$ aqueous acetone extracted higher levels of phenolic, flavonoid and condensed tannin compounds from litchi pulp than any of the other solvent systems tested. These results were similar to those reported for litchi flowers by Liu et al. [25], where the acetone extracts of litchi flowers contained the highest total amounts of phenolic, flavonoid and condensed tannin compounds of the three solvent systems tested (i.e., acetone, methanol and water). Prasad et al. [4], however, reported that $50 \%$ aqueous ethanol extracts of litchi seeds contained the highest number of total phenolic compounds and showed the highest total antioxidant capacity, following their evaluation of five different polar solvents, including ethanol, 50\% ethanol, methanol, 50\% methanol and water. The phenolic extraction efficiencies of the different parts of litchi may be dependent on the polarities of the compounds that are being extracted and different solvents may therefore be required depending on the compounds contained in the different parts of litchi. Previous studies have shown that the use of an acetone-water mixture affords much higher yields of total phenolic compounds than that of a methanolwater mixture because these extracts contain more flavan-3-ols and conjugated forms of ellagic acid [12,26]. Aqueous acetone extracts generally contain more tannins than any other extract, and similar results were observed in the current study, where the $80 \%$ aqueous acetone extract contained much more condensed tannin than any of the other extracts. Furthermore, acetone displayed higher extraction efficiency for phenolic and flavonoid compounds than any of the other solvent systems used in the current study. Thus, the acetone mixture represents a more efficient solvent system for the extraction of free phenolic compounds from litchi pulp. The free phenolic content observed in the litchi pulp in the current study was much higher than those detected in the litchi cultivars from northern Mauritius [10] and southern Florida [27], where the phenolic compounds were extracted with acetone/water $(70: 30 \mathrm{v} / \mathrm{v})$

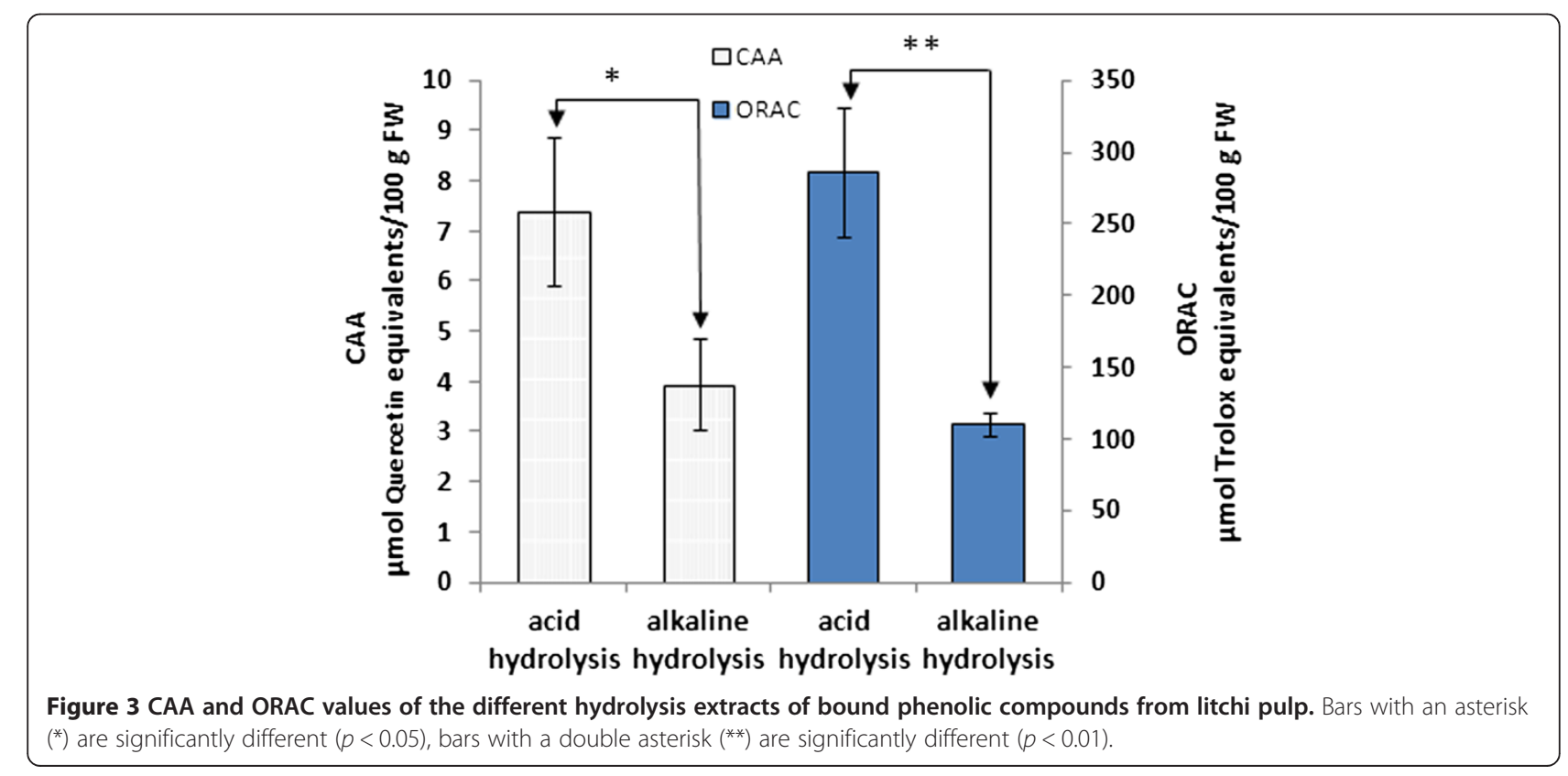


and methanol (100\%), respectively. As well as the different litchi genotypes tested, the observed discrepancies in the phenolic contents of these litchi pulps can also be attributed to differences in the solvent systems used for their extraction.

As mentioned above, there are some bound phenolic compounds in plants that cannot be extracted through solvent extraction. Enzymatic, thermal and alkaline hydrolysis methods have all been used to extract bound phenolic compounds from different sources, with alkaline hydrolysis being the most commonly used of these methods. In the current study, however, more bound phenolic, flavonoid and hydrolysed tannin compounds were obtained by acid hydrolysis than alkaline hydrolysis. As previously reported, far more bound phenolic compounds were released from apple and peach (80.3 and $52.8 \mathrm{mg}$ GAE/100 $\mathrm{g} \mathrm{FW)} \mathrm{by} \mathrm{acid} \mathrm{hydrolysis} \mathrm{[28]}$ than (4.9 and $3.2 \mathrm{mg} \mathrm{GAE} / 100 \mathrm{~g}$ FW) alkaline hydrolysis [11], because the higher temperature used for the acid hydrolysis method allowed for the release of the bound phenolic compounds trapped in the cores or conjugated to cell wall dietary fibres or proteins [14,28]. The acid hydrolysis method would therefore be more suitable than the alkaline hydrolysis method for the extraction of bound phenolic compounds from litchi pulp.

\section{Effects of different extraction solvents on the compositions and contents of phenolic compounds in litchi pulp}

The phenolic compound compositions in the free fraction were affected by the nature of the extraction solvent system. All nine compounds were found in the $80 \%$ acetone extract, whereas 3,4-dihydroxybenzoic acid and syringic acid were not detected in the $80 \%$ methanol, $80 \%$ ethyl acetate or water extracts. Furthermore, 3, 4dihydroxybenzoic acid and vanillic acid were not detected in the $80 \%$ ethanol extract. It is noteworthy that the total contents of these three acids were less than $500 \mu \mathrm{g} / 100 \mathrm{~g} \mathrm{FW}$ and therefore had little influence to the total phenolic content of the litchi pulp. The 4methylcatechol, rutin and (-)-epicatechin levels, however, varied considerably depending on the solvent system used for the extraction, with the use of $80 \%$ acetone providing the highest levels of these compounds. $M$ Kajdzanoska, J Petreska and M Stefova [12] compared a variety of different solvent mixtures for the extraction of phenolic compounds from strawberries and reported that the acetone extract contained all of the phenolic compounds that could be detected. Gallic acid, procyanidin B2 (-)-gallocatechin, (-)-epicatechin, and (-)-epicatechin-3-gallate have all been found in the pericarp $[4,29]$ and seeds [5] of litchi, although (-)-epicatechin, which serves as one of the major procyanidin compounds in litchi seeds and pericarp, was found at much higher levels $[4,5,29]$. In our previous study, we showed that rutin and (-)-epicatechin were the main free phenolic compounds in litchi pulp [19]. It is noteworthy that similar phenolic compounds exist in different parts of litchi. The most abundant phenolic compound, 4-methylcatechol, which has not been previously reported in litchi pulp, was found in the current study, and this compound can lead to elevated levels of endogenous nerve growth factor in vivo [30]. As mentioned above, the acetone extracts of litchi pulp contained higher total amounts of phenolic compounds than those of the methanol solvent system. Similar levels of 4-methylcatechol and rutin, however, were found in the aqueous acetone and methanol extracts, indicating that these solvent systems performed equally as well for the extraction of these compounds. Furthermore, the condensed tannins in the extracts were detected using a chemical method, indicating that proanthocyanidins may present in litchi pulp. Arranz et al. [28] reported the presence of extractable proanthocyanidins in the methanol/acetone extracts of apple, nectarine and peach pulp by normal-phase HPLC [28].

As shown by our previous research, four hydrolysable compounds were released from the litchi pulp residue, although seven individual phenolic compounds, including compounds generally associated with the alkaline hydrolysis of bound phenolic compounds, were detected in extracts obtained from the acid hydrolysis preparation. Ferulic acid, $p$-hydroxybenzoic acid, catechin, gallocatechin, gallic acid, and caffeic acid were identified as the main bound phenolic compounds released by the acidic hydrolysis of 26 different types of fruit that are regularly consumed as part of a typical Spanish diet [31]. Caffeic acid was identified in the litchi pulp in the current study at similar levels to those reported in the literature. The major bound phenolic compounds isolated from litchi were (-)-epicatechin and 4-methylcatechol, which could be attributed to different fruits being tested. Acidic hydrolysis represents an effective method for the release of bound phenolic compounds trapped in the cores or the fruits and bound to cell wall matrix [28]. The use of the acid hydrolysis method therefore allowed for the detection of higher levels of bound phenolic compounds and hydrolysable tannins. It is noteworthy, however, that some hydroxycinnamic acids may be lost during the acidic hydrolysis of the residues.

The phenolic compounds discussed in the current study were identified based only on their retention time by HPLC analysis and the use of other analytical methods such as HPLC-MS and NMR would be necessary to allow for the structures of these compounds to be determined beyond a reasonable doubt.

\section{Antioxidant activity of free and bound phenolic compounds of litchi pulp}

Litchi pulp contains a high level of phenolic compounds and in our previous study we found that both the free 
and bound phenolic compounds from litchi pulp exhibited excellent antioxidant activities based using DPPH and FRAP assays [19]. In the current study, the aqueous acetone extracts exhibited higher ORAC values than the methanol extracts, most likely because higher levels of (-)-epicatechin were found in the acetone extracts. The (-)-epicatechin exhibited good antioxidant activity based on the results of the ORAC assay [32]. The $80 \%$ acetone extracts therefore exhibited higher ORAC values than the $80 \%$ methanol extracts.

The CAA values for the different extracts, however, appeared in a different order to those obtained from the ORAC assay. The acetone and methanol solvent extracts had similar CAA values, which were higher than those of the other extracts. Of the three phenolic compounds, rutin exhibited no activity in the CAA assay, whereas (-)-epicatechin showed very little activity $(1.9 \mu \mathrm{mol}$ of $\mathrm{QE} / 100 \mu \mathrm{mol}$ ) [32]. Furthermore, the 4-methylcatechol contents were effectively the same in these two solvent extracts. Therefore, the main cellular antioxidant activity of the litchi pulp was attributed to 4-methylcatechol. The cellular antioxidant activities of common fruits have been reported as follows: wildberry ranked first $(292 \mu \mathrm{mol}$ of $\mathrm{QE} / 100 \mathrm{~g} \mathrm{FW}$ ), followed sequentially by blackberry (232 $\mu \mathrm{mol}$ of QE/100 g FW), raspberry $(114 \mu \mathrm{mol}$ of QE/ $100 \mathrm{~g} F W)$, cranberry (47.9 $\mu \mathrm{mol}$ of QE/100 g FW), plum $(33.5 \mu \mathrm{mol}$ of QE/100 g FW), cherry $(27.4 \mu \mathrm{mol}$ of QE/ $100 \mathrm{~g} \mathrm{FW})$, apple $(21.9 \mu \mathrm{mol}$ of QE/100 g FW), red grape $(16.3 \mu \mathrm{mol}$ of QE/100 g FW), kiwifruit $(16.1 \mu \mathrm{mol}$ of QE/ $100 \mathrm{~g}$ FW), mango $(15.3 \mu \mathrm{mol}$ of QE/100 g FW) and pineapple (14.8 $\mu \mathrm{mol}$ of QE/100 g FW) [24]. The litchi cultivar Feizixiao, which is the most popular litchi cultivar in southern China, exhibited better cellular antioxidant activity than the aforementioned fruits except wildberry, blackberry and raspberry.

The ORAC and CAA values of the bound phenolic compounds from the litchi pulp residue obtained by acid hydrolysis were higher than those obtained by alkaline hydrolysis, because the contents of the bound phenolic compounds were higher in the former case. Our previous study found that the FRAP and DPPH scavenging capacities of the bound phenolic compounds from litchi pulp were positively correlated with the phenolic and flavonoid contents [19]. It can be concluded that the litchi cultivar Feizixiao represents a good dietary antioxidant supplement fruit.

\section{Conclusions}

The free and bound phenolic compound contents, profiles and antioxidant activities of the extracts of litchi pulp were investigated and found to be significantly affected by the solvent system used for the extraction process. Of the five solvent mixtures evaluated in the current study for the extraction of the free phenolic compounds from litchi pulp, the use of an aqueous acetone mixture yielded the highest total contents of phenolic, flavonoid and tannin compounds, and exhibited the highest antioxidant activity. The use of an acid hydrolysis method resulted in higher extraction efficiency and antioxidant activity for the bound phenolic compounds in litchi pulp than an alkaline method. Nine individual phenolic compounds were detected in the aqueous acetone extract, and seven individual phenolic compounds were released by acid hydrolysis. The contents of both the free and bound individual phenolic compounds were affected by the choice of extraction solvent. Litchi pulp is rich in phenolic compounds and has good cellular antioxidant capacity.

\section{Competing interests}

The authors have declared that they have no competing interests.

\section{Authors' contributions}

MWZ conceived of this study and designed the experiments. DXS, FLH and JXG performed the experiments. All of the authors including MWZ analysed the data and discussed the results. RFZ and DXS drafted the manuscript with the help of MWZ. All of the authors have read and approved the final manuscript.

\section{Acknowledgements}

This work was supported by a Joint Fund from the NSFC and the Guangdong Provincial Government (U1301211), the Pearl River Science and Technology Star Fund (2011J2200031), the International Science \& Technology Cooperation Program of China (2012DFA31400), the National Basic Research Program of China (973 Project) (2012CB722904), the National Nature Science Foundation of China (31171680), and the Guangdong International Cooperation Project (2011B050400002).

\section{Author details}

${ }^{1}$ Department of Food Science and Technology, Huazhong Agricultura University, Wuhan 430070, P.R. China. ${ }^{2}$ Sericultural and Agri-food Research Institute, Guangdong Academy of Agricultural Sciences, Guangzhou 510610, P.R. China. ${ }^{3}$ College of Food Science, Guangdong Pharmaceutical University, Zhongshan 528458, P.R. China.

Received: 4 September 2013 Accepted: 18 December 2013 Published: 9 January 2014

\section{References}

1. Arts ICW, Hollman PCH: Polyphenols and disease risk in epidemiologic studies. Am J Clin Nutr 2005, 81(1):317S-325S.

2. Wolfe KL, Liu RH: Cellular Antioxidant Activity (CAA) assay for assessing antioxidants, foods, and dietary supplements. J Agric Food Chem 2007, 55:8896-8907.

3. Jiang YM, Duan XW, Joyce D, Zhang ZQ, Li JR: Advances in understanding of enzymatic browning in harvested litchi fruit. Food Chem 2004, 88(3):443-446.

4. Prasad KN, Yang B, Yang SY, Chen YL, Zhao MM, Ashraf M, Jiang YM: Identification of phenolic compounds and appraisal of antioxidant and antityrosinase activities from litchi (Litchi sinensis Sonn.) seeds. Food Chem 2009, 116(1):1-7.

5. Zhao MM, Yang B, Wang JS, Li BZ, Jiang YM: Identification of the major flavonoids from pericarp tissues of lychee fruit in relation to their antioxidant activities. Food Chem 2006, 98(3):539-544.

6. Zhang $Z Q$, Pang $X Q$, Yang $C$, Ji ZL, Jiang YM: Purification and structural analysis of anthocyanins from litchi pericarp. Food Chem 2004, 84(4):601-604.

7. Perez-Jimenez J, Torres JL: Analysis of nonextractable phenolic compounds in foods: the current state of the art. J Agric Food Chem 2011, 59:12713-12724. 
8. Bhoopat L, Srichairatanakool S, Kanjanapothi D, Taesotikul T, Thananchai H, Bhoopat T: Hepatoprotective effects of lychee (Litchi chinensis Sonn.): a combination of antioxidant and anti-apoptotic activities. J Ethnopharmacol 2011, 136(1):55-66.

9. Saxena S, Hajare SN, More V, Kumar S, Wadhawan S, Mishra BB, Parte MN, Gautam S, Sharma A: Antioxidant and radioprotective properties of commercially grown litchi (Litchi chinensis) from India. Food Chem 2011, 126(1):39-45.

10. Luximon-Ramma A, Bahorun T, Crozier A: Antioxidant actions and phenolic and vitamin C contents of common Mauritian exotic fruits. J SCi Food Agric 2003, 83(5):496-502.

11. Sun J, Chu YF, Wu X, Liu RH: Antioxidant and antiproliferative activities of common fruits. J Agric Food Chem 2002, 50(25):7449-7454.

12. Kajdzanoska M, Petreska J, Stefova M: Comparison of different extraction solvent mixtures for characterization of phenolic compounds in strawberries. J Agric Food Chem 2011, 59:5272-5278.

13. Ghasemzadeh A, Jaafar HZ, Rahmat A: Effects of solvent type on phenolics and flavonoids content and antioxidant activities in two varieties of young ginger (Zingiber officinale Roscoe) extracts. J Med Plants Res 2011, 5:1147-1154.

14. Bonoli M, Verardo V, Marconi E, Caboni MF: Antioxidant phenols in barley (Hordeum vulgare L.) flour: comparative spectrophotometric study among extraction methods of free and bound phenolic compounds. J Agric Food Chem 2004, 52(16):5195-5200.

15. Naczk M, Shahidi F: The effect of methanol-ammonia-water treatment on the content of phenolic acids of canola. Food Chem 1989, 31:159-164

16. Cuevas Montilla E, Hillebrand S, Antezana A, Winterhalter P: Soluble and bound phenolic compounds in different Bolivian purple corn (Zea mays L.) Cultivars. J Agric Food Chem 2011, 59(13):7068-7074

17. Madhujith T, Shahidi F: Antioxidant potential of barley as affected by alkaline hydrolysis and release of insoluble-bound phenolics. Food Chem 2009, 117(4):615-620.

18. Liyana-Pathirana CM, Shahidi F: Importance of insoluble-bound phenolics to antioxidant properties of wheat. J Agric Food Chem 2006, 54(4):1256-1264

19. Zhang R, Zeng Q, Deng Y, Zhang M, Wei Z, Zhang Y, Tang X: Phenolic profiles and antioxidant activity of litchi pulp of different cultivars cultivated in Southern China. Food Chem 2013, 136(3-4):1169-1176.

20. Liu RH: Potential synergy of phytochemicals in cancer prevention: mechanism of action. J Nutr 2004, 134(12):3479S-3485S.

21. Hartzfeld PW, Forkner R, Hunter MD, Hagerman AE: Determination of Hydrolyzable Tannins (Gallotannins and Ellagitannins) after reaction with potassium iodate. J Agric Food Chem 2002, 50:1785-1790.

22. Dewanto $V$, Wu XZ, Adom KK, Liu RH: Thermal processing enhances the nutritional value of tomatoes by increasing total antioxidant activity. $J$ Agric Food Chem 2002, 50:3010-3014.

23. Julkunen-Tiitto R: Phenolic constituents in the leaves of northern willows: methods for the analysis of certain phenolics. J Agric Food Chem 1985, 33(2):213-217.

24. Wolfe KL, Kang X, He X, Dong M, Zhang Q, Liu RH: Cellular antioxidant activity of common fruits. J Agric Food Chem 2008, 56:8418-8426.

25. Liu S-C, Lin J-T, Wang C-K, Chen H-Y, Yang D-J: Antioxidant properties of various solvent extracts from lychee. Food Chem 2009, 114:577-581.

26. Weidner S, Rybarczyk A, Karamać M, Król A, Mostek A, Grębosz J, Amarowicz $\mathrm{R}$ : Differences in the phenolic composition and Antioxidant Properties between Vitis coignetiae and Vitis vinifera seeds extracts. Molecules 2013, 18(3):3410-3426.

27. Mahattanatawee K, Manthey JA, Luzio G, Talcott ST, Goodner K, Baldwin EA: Total antioxidant activity and fiber content of select Florida-grown tropical fruits. J Agric Food Chem 2006, 54(19):7355-7363.

28. Arranz S, Saura-Calixto F, Shaha S, Kroon PA: High contents of nonextractable polyphenols in fruits suggest that polyphenol contents of plant foods have been underestimated. J Agric Food Chem 2009, 57(16):7298-7303.

29. Duan XW, Wu GF, Jiang YM: Evaluation of the antioxidant properties of litchi fruit phenolics in relation to pericarp browning prevention. Molecules 2007, 12(4):759-771.

30. Hanaoka Y, Ohi T, Furukawa S, Furukawa Y, Hayashi K, Matsukura S: Effect of 4-methylcatechol on sciatic nerve growth factor level and motor nerve conduction velocity in experimental diabetic neuropathic process in rats. Exp Neurol 1992, 115(2):292-296.

31. Arranz S, Silván JM, Saura-Calixto F: Nonextractable polyphenols, usually ignored, are the major part of dietary polyphenols: a study on the Spanish diet. Mol Nutr Food Res 2010, 54(11):1646-1658.

32. Wolfe KL, Liu RH: Structure - activity relationships of flavonoids in the cellular antioxidant activity assay. J Agric Food Chem 2008, 56(18):8404-8841.

doi:10.1186/1472-6882-14-9

Cite this article as: Su et al.: Comparison of the free and bound phenolic profiles and cellular antioxidant activities of litchi pulp extracts from different solvents. BMC Complementary and Alternative Medicine 2014 14:9.

\section{Submit your next manuscript to BioMed Central and take full advantage of:}

- Convenient online submission

- Thorough peer review

- No space constraints or color figure charges

- Immediate publication on acceptance

- Inclusion in PubMed, CAS, Scopus and Google Scholar

- Research which is freely available for redistribution 